\title{
Continuous Dependence for the Linear Differential Equations of Thermo-Diffusion
}

\author{
Jincheng Shi \\ Huashang College Guangdong University of Finance \& Economics, Guangzhou, China \\ Email: hning0818@163.com
}

How to cite this paper: Shi, J.C. (2020) Continuous Dependence for the Linear Differential Equations of Thermo-Diffusion. Journal of Applied Mathematics and Physics, 8, 1291-1303.

https://doi.org/10.4236/jamp.2020.87099

Received: June 17, 2020

Accepted: July 12, 2020

Published: July 15, 2020

Copyright (c) 2020 by author(s) and Scientific Research Publishing Inc. This work is licensed under the Creative Commons Attribution International License (CC BY 4.0).

http://creativecommons.org/licenses/by/4.0/

\begin{abstract}
In this paper, we establish the structural stability for the linear differential equations of thermo-diffusion in a semi-infinite pipe flow. Using the technology of a second-order differential inequality, we prove the continuous dependence on the density $\rho$ and the coefficient of thermal conductivity $K$. These results show that small changes for these coefficients can't cause tremendous changes for the solutions.
\end{abstract}

\section{Keywords}

Differential Equations of Thermo-Diffusion, Structural Stability, Continuous Dependence

\section{Introduction}

The question of continuous dependence of solutions of problems in partial differential equations on coefficients in the equations has been extensively studied in recent years for a variety of problems. This is sometimes referred to as the question of structural stability and numerous references may be found, for instance, in the book of Ames and Straughan [1] and the monograph of Straughan [2]. For more papers one can see [3]-[8]. In structural stability the emphasis is on continuous dependence (convergence result) on changes in the model itself rather than on the initial data. This means changes in coefficients in the partial differential equations and changes in the equations and may be reflected physically by changes in constitutive parameters. What's more, the inevitable error that arises in both numerical computation and the physical measurement of data can exist. It is relevant to know the magnitude of the effect of such errors on the solution.

In the 1970s, W. Nowacki in his papers [9] [10] gave the differential equations 
of thermodiffusion in one dimensional space and many papers in the literatures have studied this system. For example, [10] [11] [12] investigated the initial-boundary value problem for the linear system of thermodiffusion using different arguments. [13] proved the existence, uniqueness and regularity of the solution to the initial-boundary value problems for the linear system of thermodiffusion in a solid body. $\boldsymbol{L}^{p}-\boldsymbol{L}^{q}$ time decay estimates for the solution of the associated linear Cauchy problem were obtained by [14]. However, in this paper, we considered the differential equations of thermodiffusion in three dimensions and we study not only the continuous dependence on the coefficients of the equations, but also the spatial decay estimates for the solution of the system. In fact, much has been written on the subject of spatial decay bounds for various systems of differential equations, e.g., for a review of such works on Saint-Venant's principle, one can refer to [15]-[23] and the papers cited therein. Recently, there are some new results about structural stability, one could see [24]-[28].

We shall assume that a transient flow occupies the interior of a semi-infinite cylindrical pipe $\boldsymbol{R}$ with boundary $\partial \boldsymbol{R}$. The pipe has arbitrary cross section denoted by $\boldsymbol{D}$ and the boundary $\partial \boldsymbol{D}$ and the generators of the pipe are parallel to the $x_{3}$ axis. We introduce the notations:

$$
\begin{aligned}
& \boldsymbol{R}_{z}=\left\{\left(x_{1}, x_{2}, x_{3}\right) \mid\left(x_{1}, x_{2}\right) \in \boldsymbol{D}, x_{3}>z \geq 0\right\}, \\
& \boldsymbol{D}_{z}=\left\{\left(x_{1}, x_{2}, x_{3}\right) \mid\left(x_{1}, x_{2}\right) \in \boldsymbol{D}, x_{3}=z \geq 0\right\},
\end{aligned}
$$

where $z$ is a running variable along the $x_{3}$ axis. Clearly, $\boldsymbol{R}_{0}=\boldsymbol{R}$ and $\boldsymbol{D}_{0}=\boldsymbol{D}$. Let $u_{i}, \boldsymbol{T}$, and $\boldsymbol{C}$ denote the displacement, temperature, and chemical potential as independent fields, respectively. These fields depend on the space variable $\left(x_{1}, x_{2}, x_{3}\right)$ and the time variable $t$ and satisfy the following system of equations:

$$
\begin{gathered}
\rho \ddot{u}_{i}-v \Delta u_{i}-(\lambda+v) u_{j, j i}+\gamma_{1} \boldsymbol{T}_{, i}+\gamma_{2} \boldsymbol{C}_{, i}=0, \text { in } \boldsymbol{R} \times\{t \geq 0\}, \\
c \dot{\boldsymbol{T}}-K \Delta \boldsymbol{T}+\gamma_{1} \dot{u}_{i, i}+d \dot{\boldsymbol{C}}=0, \text { in } \boldsymbol{R} \times\{t \geq 0\}, \\
n \dot{\boldsymbol{C}}-M \Delta \boldsymbol{C}+\gamma_{2} \dot{u}_{i, i}+d \dot{\boldsymbol{T}}=0, \text { in } \boldsymbol{R} \times\{t \geq 0\},
\end{gathered}
$$

with the initial-boundary conditions

$$
\begin{gathered}
u_{i}=0, \quad \boldsymbol{T}=\boldsymbol{C}=0 \quad \text { on } \partial \boldsymbol{D} \times\{t \geq 0\}, \\
u_{i}=\dot{u}_{i}=0, \quad \boldsymbol{T}=\boldsymbol{C}=0 \quad \text { in } \boldsymbol{R} \times\{t=0\} . \\
u_{i}=f_{i}\left(x_{1}, x_{2}, t\right), \boldsymbol{T}=\boldsymbol{F}\left(x_{1}, x_{2}, t\right), \boldsymbol{C}=\boldsymbol{G}\left(x_{1}, x_{2}, t\right) \text { on } \boldsymbol{D}_{0} \times\{t \geq 0\}, \\
u_{i}, u_{i, j}, \boldsymbol{T}, \boldsymbol{T}_{, i}, \boldsymbol{C}, \boldsymbol{C}_{, i}, p=o\left(x_{3}^{-1}\right) \text { uniformly in } x_{1}, x_{2}, t \text { as } x_{3} \rightarrow \infty .
\end{gathered}
$$

In Equations (1.1)-(1.3), $\Delta$ is the Laplacian operator; $\rho$ represents the density; $\gamma_{1}$ and $\gamma_{2}$ are the coefficients of thermal and diffusion dilatation; $\lambda$ and $v$ are the material coefficients; $K$ is the coefficient of thermal conductivity; $M$ is the coefficient of diffusion. $n, c, d$ are the coefficients of thermodiffusion. All the above constants are positive and satisfy 


$$
c n-d^{2}>0
$$

which implies that (1.1)-(1.3) is a hyperbolic-parabolic system of partial differential equations. In the following several sections, we may use the below inequality. Let $\boldsymbol{D}$ be a plane domain $\boldsymbol{D}$ with the boundary $\partial \boldsymbol{D}$. If $w=0$ on $\partial \boldsymbol{D}$, then

$$
\int_{D} w_{, \alpha} w_{, \alpha} \mathrm{d} A \geq \lambda_{1} \int_{D} w^{2} \mathrm{~d} x,
$$

where $\lambda_{1}$ is the smallest eigenvalue of the problem

$$
\begin{gathered}
\Delta \phi+\lambda \phi=0 \text { in } \boldsymbol{D}, \\
\phi=0 \text { on } \partial \boldsymbol{D} .
\end{gathered}
$$

This inequality has been well studied (see [29] [30]). Throughout this paper, the usual summation convention is employed with repeated Latin subscripts summed from 1 to 3 and repeat Greek subscript summed from 1 to 2 . The comma is used to indicate partial differentiation, i.e. $u_{i, j}=\frac{\partial u_{i}}{\partial x_{j}}$, $\varphi_{\alpha, \alpha}=\sum_{\alpha=1}^{2} \frac{\partial \varphi_{\alpha}}{\partial x_{\alpha}}$ and $\dot{u}_{i}$ denotes $\frac{\partial u_{i}}{\partial t}$.

The paper is structured as follows: In Section 2, we derive the continuous dependence on $\rho$. Section 3 is devoted to seeking the continuous dependence on $K$.

\section{Continuous Dependence on the Parameter $\rho$}

Lemma 1. The energy $\boldsymbol{E}(z, t)$ defined in [31]

$$
\begin{aligned}
\boldsymbol{E}(z, t)= & \frac{1}{2} \rho \int_{\boldsymbol{R}_{z}} \dot{u}_{i} \dot{u}_{i} \mathrm{~d} x+\frac{v}{2} \int_{\boldsymbol{R}_{z}} u_{i, j} u_{i, j} \mathrm{~d} x+\frac{\lambda+v}{2} \int_{\boldsymbol{R}_{z}} u_{i, i}^{2} \mathrm{~d} x \\
& +K \int_{0}^{t} \int_{\boldsymbol{R}_{z}} \boldsymbol{T}_{, j} \boldsymbol{T}_{, j} \mathrm{~d} x \mathrm{~d} \eta+M \int_{0}^{t} \int_{\boldsymbol{R}_{z}} \boldsymbol{C}_{, j} \boldsymbol{C}_{, j} \mathrm{~d} x \mathrm{~d} \eta \\
& +\int_{\boldsymbol{R}_{z}}\left[\frac{\boldsymbol{C}}{2} \boldsymbol{T}^{2}+d \boldsymbol{C} \boldsymbol{T}+\frac{n}{2} C^{2}\right] \mathrm{d} x,
\end{aligned}
$$

satisfies the following estimates

$$
\boldsymbol{E}(z, t) \leq \boldsymbol{E}(0, t) \mathrm{e}^{-\frac{1}{m_{1}(t)} z} .
$$

where

$$
m_{1}(t)=\frac{\sqrt{v} t}{\sqrt{\rho}}+\frac{\sqrt{\lambda+v} t}{\sqrt{\rho}}+\frac{\gamma_{1} \sqrt{t}}{2 \sqrt{\rho \lambda_{1} K}}+\frac{\gamma_{2} \sqrt{t}}{2 \sqrt{\rho \lambda_{1} M}}+\frac{1}{2 \sqrt{\lambda_{1}}} .
$$

Proof. These results are the main results of paper [31].

Theorem 1 . The energy expression $\tilde{\varphi}(z, t)$ satisfies the following estimates:

$$
\tilde{\boldsymbol{\varphi}}(z, t) \leq \frac{2 \pi^{2}}{\rho \rho^{*}} \tilde{\boldsymbol{E}}(0, t) \mathrm{e}^{-\frac{1}{2 m_{1}(t)^{z}}}+\frac{\pi^{2} t^{2}}{\rho^{*} \rho} \tilde{\boldsymbol{E}}(0, t)\left(\mathrm{e}^{-\frac{1}{2 m_{1}(t)^{z}}}-\mathrm{e}^{-\frac{3}{2 m_{1}(t)} z}\right) .
$$

Proof. To investigate continuous dependence on $\rho$, we have to seek a bound for $\frac{\rho}{2} \int_{R_{z}} \ddot{u}_{i} \ddot{u}_{i} \mathrm{~d} x$. To do this, we first differentiate (1.1), and then multiply with 
$\ddot{u}_{i}$, integrate over the region $\boldsymbol{R}_{z} \times[0, t]$ to obtain

$$
0=\int_{0}^{t} \int_{R_{z}}\left[\rho \ddot{u}_{i}-v \Delta \dot{u}_{i}-(\lambda+v) \dot{u}_{j, j i}+\gamma_{1} \dot{\boldsymbol{T}}_{, i}+\gamma_{2} \dot{\boldsymbol{C}}_{, i}\right] \ddot{u}_{i} \mathrm{~d} x \mathrm{~d} \eta,
$$

which follows that

$$
\begin{aligned}
0= & \frac{\rho}{2} \int_{R_{z}} \ddot{u}_{i} \ddot{u}_{i} \mathrm{~d} x+v \int_{0}^{t} \int_{D_{z}} \dot{u}_{i, 3} \ddot{u}_{i} \mathrm{~d} A \mathrm{~d} \eta+\frac{v}{2} \int_{R_{z}} \dot{u}_{i, j} \dot{u}_{i, j} \mathrm{~d} x \\
& +(\lambda+v) \int_{0}^{t} \int_{D_{z}} \dot{u}_{j, j} \ddot{u}_{3} \mathrm{~d} A \mathrm{~d} \eta+\frac{\lambda+v}{2} \int_{\boldsymbol{R}_{z}} \dot{u}_{j, j}^{2} \mathrm{~d} x \\
& +\gamma_{1} \int_{0}^{t} \int_{\boldsymbol{R}_{z}} \dot{\boldsymbol{T}}_{i} \ddot{u}_{i} \mathrm{~d} x \mathrm{~d} \eta+\gamma_{2} \int_{0}^{t} \int_{\boldsymbol{R}_{z}} \dot{\boldsymbol{C}}_{, i} \ddot{u}_{i} \mathrm{~d} x \mathrm{~d} \eta,
\end{aligned}
$$

where we have supposed that $\ddot{u}_{i}$ vanish at $t=0$. Similarly, we have

$$
\begin{aligned}
0= & c \int_{0}^{t} \int_{\boldsymbol{R}_{z}} \ddot{\boldsymbol{T}} \boldsymbol{T} \mathrm{d} x \mathrm{~d} \eta-K \int_{0}^{t} \int_{\boldsymbol{R}_{z}} \Delta \ddot{\boldsymbol{T}} \dot{\boldsymbol{T}} \mathrm{d} x \mathrm{~d} \eta+\gamma_{1} \int_{0}^{t} \int_{\boldsymbol{R}_{z}} \ddot{u}_{i, i} \dot{\boldsymbol{T}} \mathrm{d} x \mathrm{~d} \eta+d \int_{0}^{t} \int_{\boldsymbol{R}_{z}} \ddot{\boldsymbol{C}} \dot{\boldsymbol{T}} \mathrm{d} x \mathrm{~d} \eta \\
= & \frac{c}{2} \int_{\boldsymbol{R}_{z}} \dot{\boldsymbol{T}}^{2} \mathrm{~d} x+K \int_{0}^{t} \int_{\boldsymbol{D}_{z}} \dot{\boldsymbol{T}_{3}} \dot{\boldsymbol{T}} \mathrm{d} A \mathrm{~d} \eta+K \int_{0}^{t} \int_{\boldsymbol{R}_{z}} \dot{\boldsymbol{T}}_{i} \dot{\boldsymbol{T}}_{, i} \mathrm{~d} x \mathrm{~d} \eta \\
& -\gamma_{1} \int_{0}^{t} \int_{\boldsymbol{D}_{z}} \ddot{\boldsymbol{u}}_{3} \dot{\boldsymbol{T}} \mathrm{d} A \mathrm{~d} \eta-\gamma_{1} \int_{0}^{t} \int_{\boldsymbol{R}_{z}} \dot{\boldsymbol{T}}_{,} \ddot{u_{i}} \mathrm{~d} x \mathrm{~d} \eta+d \int_{0}^{t} \int_{\boldsymbol{R}_{z}} \ddot{\boldsymbol{C}} \dot{\boldsymbol{T}} \mathrm{d} x \mathrm{~d} \eta,
\end{aligned}
$$

and

$$
\begin{aligned}
0= & n \int_{0}^{t} \int_{\boldsymbol{R}_{z}} \ddot{\boldsymbol{C}} \dot{\boldsymbol{C}} \mathrm{d} x \mathrm{~d} \eta-M \int_{0}^{t} \int_{\boldsymbol{R}_{z}} \Delta \dot{\boldsymbol{C}} \dot{\boldsymbol{C}} \mathrm{d} x \mathrm{~d} \eta+\gamma_{2} \int_{0}^{t} \int_{\boldsymbol{R}_{z}} \ddot{u}_{i, i} \dot{\boldsymbol{C}} \mathrm{d} x \mathrm{~d} \eta+d \int_{0}^{t} \int_{\boldsymbol{R}_{z}} \ddot{\boldsymbol{T}} \dot{\boldsymbol{C}} \mathrm{d} x \mathrm{~d} \eta \\
= & \frac{n}{2} \int_{\boldsymbol{R}_{z}} \dot{\boldsymbol{C}}^{2} \mathrm{~d} x+M \int_{0}^{t} \int_{D_{z}} \dot{\boldsymbol{C}}_{, 3} \dot{\boldsymbol{C}} \mathrm{d} A \mathrm{~d} \eta+M \int_{0}^{t} \int_{\boldsymbol{R}_{z}} \dot{\boldsymbol{C}}_{, i} \dot{\boldsymbol{C}}_{, i} \mathrm{~d} x \mathrm{~d} \eta \\
& -\gamma_{2} \int_{0}^{t} \int_{D_{z}} \ddot{\boldsymbol{u}_{3}} \dot{\boldsymbol{C}} \mathrm{d} A d \eta-\gamma_{2} \int_{0}^{t} \int_{\boldsymbol{R}_{z}} \dot{\boldsymbol{C}}_{, i} \ddot{u}_{i} \mathrm{~d} x \mathrm{~d} \eta+d \int_{0}^{t} \int_{\boldsymbol{R}_{z}} \ddot{\boldsymbol{T}} \dot{\boldsymbol{C}} \mathrm{d} x \mathrm{~d} \eta,
\end{aligned}
$$

where we also have assumed that $\dot{\boldsymbol{T}}=\dot{\boldsymbol{C}}=0$ at $t=0$. Combining (6)-(8), we set

$$
\begin{aligned}
\tilde{\boldsymbol{E}}(z, t)= & \frac{\rho}{2} \int_{\boldsymbol{R}_{z}} \ddot{u}_{i} \ddot{u}_{i} \mathrm{~d} x+\frac{v}{2} \int_{\boldsymbol{R}_{z}} \dot{u}_{i, j} \dot{u}_{i, j} \mathrm{~d} x+\frac{\lambda+v}{2} \int_{\boldsymbol{R}_{z}} \dot{u}_{j, j}^{2} \mathrm{~d} x+K \int_{0}^{t} \int_{\boldsymbol{R}_{z}} \dot{\boldsymbol{T}}_{, i} \dot{\boldsymbol{T}}_{, i} \mathrm{~d} x \mathrm{~d} \eta \\
& +M \int_{0}^{t} \int_{\boldsymbol{R}_{z}} \dot{\boldsymbol{C}}_{, i} \dot{\boldsymbol{C}}_{, i} \mathrm{~d} x \mathrm{~d} \eta+\frac{c}{2} \int_{\boldsymbol{R}_{z}} \dot{\boldsymbol{T}}^{2} \mathrm{~d} x+\frac{1}{2} \int_{\boldsymbol{R}_{z}}\left[c \dot{\boldsymbol{T}}^{2}+2 d \dot{\boldsymbol{T}} \dot{\boldsymbol{C}}+n \dot{\boldsymbol{C}}^{2}\right] \mathrm{d} x \\
= & -v \int_{0}^{t} \int_{D_{z}} \dot{u}_{i, 3} \ddot{u}_{i} \mathrm{~d} A \mathrm{~d} \eta-(\lambda+v) \int_{0}^{t} \int_{D_{z}} \dot{u}_{j, j} \ddot{u}_{3} \mathrm{~d} A \mathrm{~d} \eta-K \int_{0}^{t} \int_{D_{z}} \dot{\boldsymbol{T}}_{, 3} \dot{\boldsymbol{T}} \mathrm{d} A \mathrm{~d} \eta \\
& +\gamma_{1} \int_{0}^{t} \int_{D_{z}} \ddot{u}_{3} \dot{\boldsymbol{T}} \mathrm{d} A \mathrm{~d} \eta-M \int_{0}^{t} \int_{D_{z}} \dot{\boldsymbol{C}}_{, 3} \dot{\boldsymbol{C}} \mathrm{d} A \mathrm{~d} \eta+\gamma_{2} \int_{0}^{t} \int_{D_{z}} \ddot{u}_{3} \dot{\boldsymbol{C}} \mathrm{d} A \mathrm{~d} \eta .
\end{aligned}
$$

Following the method used in [31], we can get

$$
\begin{aligned}
\tilde{\boldsymbol{E}}(z, t)= & \frac{\rho}{2} \int_{\boldsymbol{R}_{z}} \ddot{u}_{i} \ddot{u}_{i} \mathrm{~d} x+\frac{v}{2} \int_{\boldsymbol{R}_{z}} \dot{u}_{i, j} \dot{u}_{i, j} \mathrm{~d} x+\frac{\lambda+v}{2} \int_{\boldsymbol{R}_{z}} \dot{u}_{j, j}^{2} \mathrm{~d} x+K \int_{0}^{t} \int_{\boldsymbol{R}_{z}} \dot{\boldsymbol{T}}_{, i} \dot{\boldsymbol{T}}_{, i} \mathrm{~d} x \mathrm{~d} \eta \\
& +M \int_{0}^{t} \int_{\boldsymbol{R}_{z}} \dot{\boldsymbol{C}}_{, i} \dot{\boldsymbol{C}}_{, i} \mathrm{~d} x \mathrm{~d} \eta+\frac{c}{2} \int_{\boldsymbol{R}_{z}} \dot{\boldsymbol{T}}^{2} \mathrm{~d} x+\frac{1}{2} \int_{\boldsymbol{R}_{z}}\left[c \dot{\boldsymbol{T}}^{2}+2 d \dot{\boldsymbol{T}} \dot{\boldsymbol{C}}+n \dot{\boldsymbol{C}}^{2}\right] \mathrm{d} x \\
\leq & \tilde{\boldsymbol{E}}(0, t) \mathrm{e}^{\left.-\frac{1}{m_{1}(t)}\right)^{z}} .
\end{aligned}
$$

Also, we employ the argument used in [31] to get that $\tilde{\boldsymbol{E}}(0, t)$ may be bounded by known data. Since $c n>d^{2}$, we note again

$\int_{R_{z}}\left[c \dot{\boldsymbol{T}}^{2}+2 d \dot{\boldsymbol{T}} \dot{\boldsymbol{C}}+n \dot{\boldsymbol{C}}^{2}\right] \mathrm{d} x>0$. So, we have

$$
\int_{R_{z}} \ddot{u}_{i} \ddot{u}_{i} \mathrm{~d} x \leq \frac{2}{\rho} \tilde{\boldsymbol{E}}(0, t) \mathrm{e}^{-\frac{1}{m_{1}(t)}} .
$$

Now, we study the continuous dependence on the parameter $\rho$. Let $\left(u_{i}, \boldsymbol{T}, \boldsymbol{C}\right)$ 
and $\left(u_{i}^{*}, \boldsymbol{T}^{*}, \boldsymbol{C}^{*}\right)$ be the solutions to (1.1)-(1.3) with same initial-boundary conditions, but for different parameters $\rho$ and $\rho^{*}$, respectively. Define the difference variables as

$$
w_{i}=u_{i}-u_{i}^{*}, \quad \theta=\boldsymbol{T}-\boldsymbol{T}^{*}, \quad \Sigma=\boldsymbol{C}-\boldsymbol{C}^{*}, \quad \pi=\rho-\rho^{*} .
$$

Then, $\left(w_{i}, \theta, \Sigma\right)$ satisfy

$$
\begin{gathered}
\pi \ddot{u}_{i}+\rho^{*} \ddot{w}_{i}-v \Delta w_{i}-(\lambda+v) w_{j, j i}+\gamma_{1} \theta_{, i}+\gamma_{2} \Sigma_{, i}=0, \text { in } \boldsymbol{R} \times\{t \geq 0\}, \\
c \dot{\theta}-K \Delta \theta+\gamma_{1} \dot{w}_{i, i}+d \dot{\Sigma}=0, \text { in } \boldsymbol{R} \times\{t \geq 0\}, \\
n \dot{\Sigma}-M \Delta \Sigma+\gamma_{2} \dot{w}_{i, i}+d \dot{\theta}=0, \text { in } \boldsymbol{R} \times\{t \geq 0\},
\end{gathered}
$$

with the initial-boundary conditions

$$
\begin{gathered}
w_{i}=0, \quad \theta=\Sigma=0 \quad \text { on } \partial \boldsymbol{D} \times\{t \geq 0\}, \\
w_{i}=\dot{w}_{i}=0, \quad \theta=\Sigma=0 \quad \text { in } \boldsymbol{R} \times\{t=0\} . \\
w_{i}=\theta=\Sigma=0 \quad \text { on } \boldsymbol{D}_{0} \times\{t \geq 0\},
\end{gathered}
$$

Multiplying (2.12) with $\dot{w}_{i}$ and integrating by parts, we have

$$
\begin{aligned}
0= & \int_{0}^{t} \int_{R_{z}}\left[\pi \ddot{u}_{i}+\rho^{*} \ddot{w}_{i}-v \Delta w_{i}-(\lambda+v) w_{j, j i}+\gamma_{1} \theta_{, i}+\gamma_{2} \Sigma_{, i}\right] \dot{w}_{i} \mathrm{~d} x \mathrm{~d} \eta \\
= & \frac{1}{2} \int_{R_{z}} \rho^{*} \dot{w}_{i} \dot{w}_{i} d x+\frac{v}{2} \int_{R_{z}} w_{i, j} w_{i, j} \mathrm{~d} x+\frac{\lambda+v}{2} \int_{R_{z}} w_{i, i}^{2} \mathrm{~d} x \\
& +\pi \int_{0}^{t} \int_{R_{z}} \ddot{u}_{i} \dot{w}_{i} \mathrm{~d} x \mathrm{~d} \eta+\gamma_{1} \int_{0}^{t} \int_{R_{z}} \theta_{, i} \dot{w}_{i} \mathrm{~d} x \mathrm{~d} \eta+\gamma_{2} \int_{0}^{t} \int_{R_{z}} \Sigma_{, i} \dot{w}_{i} \mathrm{~d} x \mathrm{~d} \eta \\
& +v \int_{0}^{t} \int_{D_{z}} w_{i, 3} \dot{w}_{i} \mathrm{~d} A \mathrm{~d} \eta+(\lambda+v) \int_{0}^{t} \int_{D_{z}} w_{j, j} \dot{w}_{3} \mathrm{~d} A \mathrm{~d} \eta .
\end{aligned}
$$

Similarly, we have

$$
\begin{aligned}
0= & \int_{0}^{t} \int_{R_{z}}\left[c \dot{\theta}-K \Delta \theta+\gamma_{1} w_{i, i}+d \dot{\Sigma}\right] \theta \mathrm{d} x \mathrm{~d} \eta \\
= & \frac{c}{2} \int_{R_{z}} \theta^{2} \mathrm{~d} x \mathrm{~d} \eta+K \int_{0}^{t} \int_{R_{z}} \theta_{, i} \theta_{, i} \mathrm{~d} x \mathrm{~d} \eta-\gamma_{1} \int_{0}^{t} \int_{R_{z}} \theta_{, i} \dot{w}_{i} \mathrm{~d} x \mathrm{~d} \eta \\
& +d \int_{0}^{t} \int_{R_{z}} \dot{\Sigma} \theta \mathrm{d} x \mathrm{~d} \eta+K \int_{0}^{t} \int_{D_{z}} \theta_{, 3} \theta \mathrm{d} A \mathrm{~d} \eta-\gamma_{1} \int_{0}^{t} \int_{D_{z}} \dot{w}_{3} \theta \mathrm{d} A \mathrm{~d} \eta,
\end{aligned}
$$

and

$$
\begin{aligned}
0= & \int_{0}^{t} \int_{R_{z}}\left[n \dot{\Sigma}-M \Delta \Sigma+\gamma_{2} w_{i, i}+d \dot{\theta}\right] \Sigma \mathrm{d} x \\
= & \frac{n}{2} \int_{\boldsymbol{R}_{z}} \Sigma^{2} \mathrm{~d} x \mathrm{~d} \eta+M \int_{0}^{t} \int_{R_{z}} \Sigma_{, i} \Sigma_{, i} \mathrm{~d} x \mathrm{~d} \eta-\gamma_{2} \int_{0}^{t} \int_{\boldsymbol{R}_{z}} \Sigma_{, i} \dot{i}_{i} \mathrm{~d} x \mathrm{~d} \eta \\
& +d \int_{0}^{t} \int_{\boldsymbol{R}_{z}} \dot{\theta} \Sigma \mathrm{d} x \mathrm{~d} \eta+M \int_{0}^{t} \int_{D_{z}} \Sigma_{, 3} \Sigma \mathrm{d} A \mathrm{~d} \eta-\gamma_{2} \int_{0}^{t} \int_{D_{z}} \dot{W}_{3} \Sigma \mathrm{d} A \mathrm{~d} \eta .
\end{aligned}
$$

We define a new function

$$
\begin{aligned}
\varphi(z, t)= & \frac{1}{2} \int_{R_{z}} \rho^{*} \dot{w}_{i} \dot{w}_{i} \mathrm{~d} x+\frac{v}{2} \int_{R_{z}} w_{i, j} w_{i, j} \mathrm{~d} x+\frac{\lambda+v}{2} \int_{R_{z}} w_{i, i}^{2} \mathrm{~d} x+K \int_{0}^{t} \int_{R_{z}} \theta_{, i} \theta_{, i} \mathrm{~d} x \mathrm{~d} \eta \\
& +M \int_{0}^{t} \int_{R_{z}} \Sigma_{, i} \Sigma_{, i} \mathrm{~d} x \mathrm{~d} \eta+\frac{1}{2} \int_{R_{z}}\left[c \theta^{2}+2 d \theta \Sigma+n \Sigma^{2}\right] \mathrm{d} x \mathrm{~d} \eta \\
= & \pi \int_{0}^{t} \int_{R_{z}} \ddot{u}_{i} \dot{w}_{i} \mathrm{~d} x \mathrm{~d} \eta-v \int_{0}^{t} \int_{D_{z}} w_{i, 3} \dot{w}_{i} \mathrm{~d} A \mathrm{~d} \eta-(\lambda+v) \int_{0}^{t} \int_{D_{z}} w_{j, j} \dot{w}_{3} \mathrm{~d} A \mathrm{~d} \eta \\
& -K \int_{0}^{t} \int_{D_{z}} \theta_{, 3} \theta \mathrm{d} A \mathrm{~d} \eta+\gamma_{1} \int_{0}^{t} \int_{D_{z}} \dot{w}_{3} \theta \mathrm{d} A \mathrm{~d} \eta \\
& -M \int_{0}^{t} \int_{D_{z}} \Sigma_{, 3} \Sigma \mathrm{d} A \mathrm{~d} \eta+\gamma_{2} \int_{0}^{t} \int_{D_{z}} \dot{w}_{3} \Sigma \mathrm{d} A \mathrm{~d} \eta .
\end{aligned}
$$


Thus, we have

$$
\begin{aligned}
-\frac{\partial \varphi(z, t)}{\partial z}= & \frac{1}{2} \int_{D_{z}} \rho^{*} \dot{w}_{i} \dot{w}_{i} \mathrm{~d} A+\frac{v}{2} \int_{D_{z}} w_{i, j} w_{i, j} \mathrm{~d} A+\frac{\lambda+v}{2} \int_{D_{z}} w_{i, i}^{2} \mathrm{~d} A \\
& +K \int_{0}^{t} \int_{D_{z}} \theta_{, i} \theta_{, i} \mathrm{~d} A \mathrm{~d} \eta+M \int_{0}^{t} \int_{D_{z}} \Sigma_{, i} \Sigma_{, i} \mathrm{~d} A \mathrm{~d} \eta \\
& +\frac{1}{2} \int_{D_{z}}\left[c \theta^{2}+2 d \theta \Sigma+n \Sigma^{2}\right] \mathrm{d} A \mathrm{~d} \eta .
\end{aligned}
$$

Similar to [31], we have

$$
\varphi(z, t) \leq \pi \int_{0}^{t} \int_{R_{z}} \ddot{u}_{i} \dot{w}_{i} \mathrm{~d} x \mathrm{~d} \eta+m_{1}(t)\left(-\frac{\partial \varphi(z, t)}{\partial z}\right),
$$

where $m_{1}(t)$ have been defined in (2.3), but $\rho$ in $m_{1}(t)$ may be replaced by $\rho^{*}$ here. By Hölder and the AG mean inequalities, from (2.23) we have

$$
\begin{aligned}
\varphi(z, t) & \leq \pi\left(\int_{0}^{t} \int_{R_{z}} \ddot{u}_{i} \ddot{u}_{i} \mathrm{~d} x \mathrm{~d} \eta\right)^{\frac{1}{2}}\left(\int_{0}^{t} \int_{R_{z}} \dot{w}_{i} \dot{w}_{i} \mathrm{~d} x \mathrm{~d} \eta\right)^{\frac{1}{2}}+m_{1}(t)\left(-\frac{\partial \varphi(z, t)}{\partial z}\right) \\
& \leq \frac{\pi^{2} t}{\rho^{*}} \int_{0}^{t} \int_{R_{z}} \ddot{u}_{i} \ddot{u}_{i} \mathrm{~d} x \mathrm{~d} \eta+\frac{\rho^{*}}{4} \int_{R_{z}} \dot{w}_{i} \dot{w}_{i} \mathrm{~d} x+m_{1}(t)\left(-\frac{\partial \varphi(z, t)}{\partial z}\right) .
\end{aligned}
$$

Now, we let

$$
\begin{aligned}
\tilde{\varphi}(z, t)= & \frac{1}{4} \int_{R_{z}} \rho^{*} \dot{w}_{i} \dot{w}_{i} \mathrm{~d} x+\frac{v}{2} \int_{R_{z}} w_{i, j} w_{i, j} \mathrm{~d} x+\frac{\lambda+v}{2} \int_{R_{z}} w_{i, i}^{2} \mathrm{~d} x \\
& +K \int_{0}^{t} \int_{R_{z}} \theta_{, i} \theta_{, i} \mathrm{~d} x \mathrm{~d} \eta+M \int_{0}^{t} \int_{\boldsymbol{R}_{z}} \Sigma_{, i} \Sigma, i d x \mathrm{~d} \eta \\
& +\frac{1}{2} \int_{R_{z}}\left[c \theta^{2}+2 d \theta \Sigma+n \Sigma^{2}\right] \mathrm{d} x \mathrm{~d} \eta .
\end{aligned}
$$

From (2.24) and (2.10), we have

$$
\tilde{\boldsymbol{\varphi}}(z, t) \leq \frac{\pi^{2} t}{\rho^{*}} \int_{0}^{t} \int_{R_{z}} \ddot{u}_{i} \ddot{u}_{i} \mathrm{~d} x \mathrm{~d} \eta+2 m_{1}(t)\left(-\frac{\partial \tilde{\varphi}(z, t)}{\partial z}\right),
$$

which follows that

$$
\tilde{\boldsymbol{\varphi}}(z, t) \leq \tilde{\boldsymbol{\varphi}}(0, t) \mathrm{e}^{-\frac{1}{2 m_{1}(t)} z}+\frac{\pi^{2} t^{2}}{\rho^{*} \rho} \tilde{\boldsymbol{E}}(0, t)\left(1-\mathrm{e}^{-\frac{1}{m_{1}(t)} z}\right) \mathrm{e}^{-\frac{1}{2 m_{1}(t)} z} .
$$

In order to make inequality (2.26) explicit, we need bound for $\tilde{\varphi}(0, t)$. So, we write (2.21) at $z=0$ and use the initial-boundary conditions to obtain

$$
\begin{aligned}
\varphi(0, t)= & \frac{1}{2} \int_{R_{0}} \rho^{*} \dot{w}_{i} \dot{w}_{i} \mathrm{~d} x+\frac{v}{2} \int_{R_{0}} w_{i, j} w_{i, j} \mathrm{~d} x+\frac{\lambda+v}{2} \int_{R_{0}} w_{i, i}^{2} \mathrm{~d} x \\
& +K \int_{0}^{t} \int_{R_{0}} \theta_{, i} \theta_{, i} \mathrm{~d} x \mathrm{~d} \eta+M \int_{0}^{t} \int_{R_{0}} \Sigma_{, i} \Sigma_{, i} \mathrm{~d} x \mathrm{~d} \eta \\
& +\frac{1}{2} \int_{R_{0}}\left[c \theta^{2}+2 d \theta \Sigma+n \Sigma^{2}\right] \mathrm{d} x \mathrm{~d} \eta \\
= & \pi \int_{0}^{t} \int_{R_{0}} \ddot{u}_{i} \dot{w}_{i} \mathrm{~d} x \mathrm{~d} \eta \\
\leq & \frac{\pi^{2} t}{\rho^{*}} \int_{0}^{t} \int_{R_{0}} \ddot{u}_{i} \ddot{u}_{i} \mathrm{~d} x \mathrm{~d} \eta+\frac{\rho^{*}}{4} \int_{R_{0}} \dot{w}_{i} \dot{w}_{i} \mathrm{~d} x,
\end{aligned}
$$

which results in 


$$
\tilde{\boldsymbol{\varphi}}(0, t) \leq \frac{\pi^{2} t}{\rho^{*}} \int_{0}^{t} \int_{R_{0}} \ddot{u}_{i} \ddot{u}_{i} \mathrm{~d} x \mathrm{~d} \eta \leq \frac{2 \pi^{2}}{\rho \rho^{*}} \tilde{\boldsymbol{E}}(0, t),
$$

where we have used (2.10). Combining (2.27) and (2.29), we have

$$
\tilde{\boldsymbol{\varphi}}(z, t) \leq \frac{2 \pi^{2}}{\rho \rho^{*}} \tilde{\boldsymbol{E}}(0, t) \mathrm{e}^{-\frac{1}{2 m_{1}(t)} z}+\frac{\pi^{2} t^{2}}{\rho^{*} \rho} \tilde{\boldsymbol{E}}(0, t)\left(\mathrm{e}^{\left.-\frac{1}{2 m_{1}(t)}\right)^{2}}-\mathrm{e}^{-\frac{3}{2 m_{1}(t)} z}\right) .
$$

Inequality (2.30) shows that the amplitude terms in (2.25) become small as $\rho \rightarrow \rho^{*}$ and the continuous dependence on $\rho$ is obtained.

\section{Continuous Dependence on the Parameter $K$}

Theorem 2. The energy expression $\tilde{\Phi}\left(z, t_{1}\right)$ satisfies the following estimates: If $h_{0}-k_{1}-k_{2}=0$, we have

$$
\tilde{\boldsymbol{\Phi}}\left(z, t_{1}\right) \leq \beta^{2} m_{4} \mathrm{e}^{-\left(h_{0}-k_{1}\right) z}+\beta^{2} m_{3} \mathrm{e}^{-\left(h_{0}-k_{1}\right) z} .
$$

If $h_{0}-k_{1}-k_{2} \neq 0$, we have

$$
\tilde{\boldsymbol{\Phi}}\left(z, t_{1}\right) \leq \beta^{2}\left[m_{4}-\frac{m_{3}}{h_{0}-k_{1}-k_{2}}\right] \mathrm{e}^{-\left(h_{0}-k_{1}\right) z}+\frac{\beta^{2} m_{3}}{h_{0}-k_{1}-k_{2}} \mathrm{e}^{-k_{2} z} .
$$

Proof. In this section we compare the solutions of the following two problems

$$
\begin{array}{ll}
\rho \ddot{u}_{i}-v \Delta u_{i}-(\lambda+v) u_{j, j i}+\gamma_{1} \boldsymbol{T}_{, i}+\gamma_{2} \boldsymbol{C}_{, i}=0, & \text { in } \boldsymbol{R} \times\{t \geq 0\}, \\
c \dot{\boldsymbol{T}}-K \Delta \boldsymbol{T}+\gamma_{1} \dot{u}_{i, i}+d \dot{\boldsymbol{C}}=0, & \text { in } \boldsymbol{R} \times\{t \geq 0\}, \\
n \dot{\boldsymbol{C}}-M \Delta \boldsymbol{C}+\gamma_{2} \dot{u}_{i, i}+d \dot{\boldsymbol{T}}=0, & \text { in } \boldsymbol{R} \times\{t \geq 0\},
\end{array}
$$

with the initial-boundary conditions

$$
\begin{array}{ll}
u_{i}=0, \quad \boldsymbol{T}=\boldsymbol{C}=0 & \text { on } \partial \boldsymbol{D} \times\{t \geq 0\}, \\
u_{i}=\dot{u}_{i}=0, \quad \boldsymbol{T}=\boldsymbol{C}=0 & \text { in } \boldsymbol{R} \times\{t=0\}, \\
u_{i}=f_{i}\left(x_{1}, x_{2}, t\right), \boldsymbol{T}=\boldsymbol{F}\left(x_{1}, x_{2}, t\right), \boldsymbol{C}=\boldsymbol{G}\left(x_{1}, x_{2}, t\right) & \text { on } \boldsymbol{D}_{0} \times\{t \geq 0\},
\end{array}
$$

and

$$
\begin{array}{ll}
\rho \ddot{u}_{i}^{*}-v \Delta u_{i}^{*}-(\lambda+v) u_{j, j i}^{*}+\gamma_{1} \boldsymbol{T}_{, i}^{*}+\gamma_{2} \boldsymbol{C}_{, i}^{*}=0, & \text { in } \boldsymbol{R} \times\{t \geq 0\}, \\
c \dot{\boldsymbol{T}}^{*}-K^{*} \Delta \boldsymbol{T}^{*}+\gamma_{1} \dot{u}_{i, i}^{*}+d \dot{\boldsymbol{C}}^{*}=0, & \text { in } \boldsymbol{R} \times\{t \geq 0\}, \\
n \dot{\boldsymbol{C}}^{*}-M \Delta \boldsymbol{C}^{*}+\gamma_{2} \dot{u}_{i, i}^{*}+d \dot{\boldsymbol{T}}^{*}=0, & \text { in } \boldsymbol{R} \times\{t \geq 0\},
\end{array}
$$

with the same initial-boundary conditions (3.4).

Our goal in this section is to derive the continuous dependence on the parameter $K$. If we define

$$
w_{i}=u_{i}-u_{i}^{*}, \quad \theta=\boldsymbol{T}-\boldsymbol{T}^{*}, \Sigma=\boldsymbol{C}-\boldsymbol{C}^{*}, \beta=K-K^{*},
$$

then, $\left(w_{i}, \theta, \Sigma\right)$ satisfy the system

$$
\begin{array}{ll}
\rho \ddot{w}_{i}-v \Delta w_{i}-(\lambda+v) w_{j, j i}+\gamma_{1} \theta_{, i}+\gamma_{2} \Sigma_{, i}=0, & \text { in } \boldsymbol{R} \times\{t \geq 0\}, \\
c \dot{\theta}-\beta \Delta \boldsymbol{T}-K^{*} \Delta \theta+\gamma_{1} \dot{w}_{i, i}+d \dot{\Sigma}=0, & \text { in } \boldsymbol{R} \times\{t \geq 0\}, \\
n \dot{\Sigma}-M \Delta \Sigma+\gamma_{2} \dot{w}_{i, i}+d \dot{\theta}=0, & \text { in } \boldsymbol{R} \times\{t \geq 0\},
\end{array}
$$

with the initial-boundary conditions 


$$
\begin{array}{ll}
w_{i}=0, \quad \theta=\Sigma=0 & \text { on } \partial \boldsymbol{D} \times\{t \geq 0\}, \\
w_{i}=\dot{w}_{i}=0, \quad \theta=\Sigma=0 & \text { in } \boldsymbol{R} \times\{t=0\}, \\
w_{i}=0, \quad \theta=\Sigma=0 & \text { on } \boldsymbol{D}_{0} \times\{t \geq 0\} .
\end{array}
$$

We multiply (3.7) $)_{1}$ with $w_{i}$ and integrate by parts to have

$$
\begin{aligned}
0= & \int_{0}^{t} \int_{R_{z}}\left[\rho \ddot{w}_{i}-v \Delta w_{i}-(\lambda+v) w_{j, j i}+\gamma_{1} \theta_{, i}+\gamma_{2} \Sigma_{, i}\right] \dot{w}_{i} \mathrm{~d} x \mathrm{~d} \eta \\
= & \frac{\rho}{2} \int_{R_{z}} \dot{w}_{i} \dot{w}_{i} \mathrm{~d} x+\frac{v}{2} \int_{R_{z}} w_{i, j} w_{i, j} \mathrm{~d} x+\frac{\lambda+v}{2} \int_{R_{z}} w_{i, i}^{2} \mathrm{~d} x+\gamma_{1} \int_{0}^{t} \int_{R_{z}} \theta_{, i} \dot{w}_{i} \mathrm{~d} x \mathrm{~d} \eta \\
& +\gamma_{2} \int_{0}^{t} \int_{R_{z}} \Sigma_{i} \dot{w}_{i} \mathrm{~d} x \mathrm{~d} \eta+v \int_{0}^{t} \int_{D_{z}} w_{i, 3} \dot{w}_{i} \mathrm{~d} A \mathrm{~d} \eta+(\lambda+v) \int_{0}^{t} \int_{D_{z}} w_{j, j} \dot{w}_{3} \mathrm{~d} A \mathrm{~d} \eta .
\end{aligned}
$$

Similarly, we have

$$
\begin{aligned}
0= & \int_{0}^{t} \int_{\boldsymbol{R}_{z}}\left[c \dot{\theta}-\beta \Delta \boldsymbol{T}-K^{*} \Delta \theta+\gamma_{1} w_{i, i}+d \dot{\Sigma}\right] \theta \mathrm{d} x \mathrm{~d} \eta \\
= & \frac{c}{2} \int_{\boldsymbol{R}_{z}} \theta^{2} \mathrm{~d} x \mathrm{~d} \eta+\beta \int_{0}^{t} \int_{\boldsymbol{R}_{z}} T_{i} \theta_{, i} \mathrm{~d} x \mathrm{~d} \eta+K^{*} \int_{0}^{t} \int_{\boldsymbol{R}_{z}} \theta_{, i} \theta_{, i} \mathrm{~d} x \mathrm{~d} \eta \\
& -\gamma_{1} \int_{0}^{t} \int_{\boldsymbol{R}_{z}} \theta_{, i} \dot{w}_{i} \mathrm{~d} x \mathrm{~d} \eta+d \int_{0}^{t} \int_{R_{z}} \dot{\Sigma} \theta \mathrm{d} x \mathrm{~d} \eta+\beta \int_{0}^{t} \int_{D_{z}} \boldsymbol{T}_{, 3} \theta \mathrm{d} A \mathrm{~d} \eta \\
& +K^{*} \int_{0}^{t} \int_{D_{z}} \theta_{3} \theta \mathrm{d} A \mathrm{~d} \eta-\gamma_{1} \int_{0}^{t} \int_{D_{z}} \dot{w}_{3} \theta \mathrm{d} A \mathrm{~d} \eta .
\end{aligned}
$$

Combining (2.20), (3.9) and (3.10), we have

$$
\begin{aligned}
\Phi(z, t) \doteq & \frac{\rho}{2} \int_{R_{z}} \dot{w}_{i} \dot{w}_{i} \mathrm{~d} x+\frac{v}{2} \int_{R_{z}} w_{i, j} w_{i, j} \mathrm{~d} x+\frac{\lambda+v}{2} \int_{\boldsymbol{R}_{z}} w_{i, i}^{2} \mathrm{~d} x+K^{*} \int_{0}^{t} \int_{\boldsymbol{R}_{z}} \theta_{, i} \theta_{, i} \mathrm{~d} x \mathrm{~d} \eta \\
& +M \int_{0}^{t} \int_{\boldsymbol{R}_{z}} \Sigma_{, i} \Sigma_{, i} \mathrm{~d} x \mathrm{~d} \eta+\frac{1}{2} \int_{\boldsymbol{R}_{z}}\left[c \theta^{2}+2 d \theta \Sigma+n \Sigma^{2}\right] \mathrm{d} x \mathrm{~d} \eta \\
= & -v \int_{0}^{t} \int_{D_{z}} w_{i, 3} \dot{w}_{i} \mathrm{~d} A \mathrm{~d} \eta-(\lambda+v) \int_{0}^{t} \int_{D_{z}} w_{j, j} \dot{W}_{3} \mathrm{~d} A \mathrm{~d} \eta-K^{*} \int_{0}^{t} \int_{D_{z}} \theta_{, 3} \theta \mathrm{d} A \mathrm{~d} \eta \\
& +\gamma_{1} \int_{0}^{t} \int_{D_{z}} \dot{W}_{3} \theta \mathrm{d} A \mathrm{~d} \eta-M \int_{0}^{t} \int_{D_{z}} \Sigma_{, 3} \Sigma \mathrm{d} A \mathrm{~d} \eta+\gamma_{2} \int_{0}^{t} \int_{D_{z}} \dot{W}_{3} \Sigma \mathrm{d} A \mathrm{~d} \eta \\
& -\beta \int_{0}^{t} \int_{D_{z}} \boldsymbol{T}_{, 3} \theta \mathrm{d} A \mathrm{~d} \eta-\beta \int_{0}^{t} \int_{\boldsymbol{R}_{z}} \boldsymbol{T}_{, i} \theta_{, i} \mathrm{~d} x \mathrm{~d} \eta .
\end{aligned}
$$

Now, we define a new function

$$
\begin{aligned}
\Gamma(z, t)= & \int_{z}^{\infty} \Phi(\xi, t) \mathrm{d} \xi \\
= & \frac{\rho}{2} \int_{R_{z}}(\xi-z) \dot{w}_{i} \dot{w}_{i} \mathrm{~d} x+\frac{v}{2} \int_{R_{z}}(\xi-z) w_{i, j} w_{i, j} \mathrm{~d} x+\frac{\lambda+v}{2} \int_{R_{z}}(\xi-z) w_{i, i}^{2} \mathrm{~d} x \\
& +K^{*} \int_{0}^{t} \int_{R_{z}}(\xi-z) \theta_{i} \theta_{, i} \mathrm{~d} x \mathrm{~d} \eta+M \int_{0}^{t} \int_{R_{z}}(\xi-z) \Sigma_{, i} \Sigma_{, i} \mathrm{~d} x \mathrm{~d} \eta \\
& +\frac{1}{2} \int_{R_{z}}(\xi-z)\left[c \theta^{2}+2 d \theta \Sigma+n \Sigma^{2}\right] \mathrm{d} x .
\end{aligned}
$$

From (3.11), we have

$$
\begin{aligned}
\Gamma(z, t)= & -v \int_{0}^{t} \int_{\boldsymbol{R}_{z}} w_{i, 3} \dot{w}_{i} \mathrm{~d} x \mathrm{~d} \eta+(\lambda+v) \int_{0}^{t} \int_{\boldsymbol{R}_{z}} w_{j, j} \dot{w}_{3} \mathrm{~d} x \mathrm{~d} \eta-K^{*} \int_{0}^{t} \int_{\boldsymbol{R}_{z}} \theta_{, 3} \theta \mathrm{d} x \mathrm{~d} \eta \\
& +\gamma_{1} \int_{0}^{t} \int_{\boldsymbol{R}_{z}} \dot{w}_{3} \theta \mathrm{d} x \mathrm{~d} \eta-M \int_{0}^{t} \int_{\boldsymbol{R}_{z}} \Sigma{ }_{, 3} \Sigma \mathrm{d} x \mathrm{~d} \eta+\gamma_{2} \int_{0}^{t} \int_{\boldsymbol{R}_{z}} \dot{W}_{3} \Sigma \mathrm{d} x \mathrm{~d} \eta \\
& -\beta \int_{0}^{t} \int_{\boldsymbol{R}_{z}} \boldsymbol{T}_{3} \theta \mathrm{d} x \mathrm{~d} \eta-\beta \int_{0}^{t} \int_{\boldsymbol{R}_{z}}(\xi-z) \boldsymbol{T}_{, i} \theta_{, i} \mathrm{~d} x \mathrm{~d} \eta .
\end{aligned}
$$

Following the same procedures which have been used in Section 3 (see 
(3.6)-(3.10)), we have

$$
\Gamma(z, t) \leq m_{1}(t)\left(-\frac{\partial \Gamma}{\partial z}(z, t)\right)-\beta \int_{0}^{t} \int_{\boldsymbol{R}_{z}} \boldsymbol{T}_{, 3} \theta \mathrm{d} x \mathrm{~d} \eta-\beta \int_{0}^{t} \int_{\boldsymbol{R}_{z}}(\xi-z) \boldsymbol{T}_{, i} \theta_{, i} \mathrm{~d} x \mathrm{~d} \eta .
$$

By Hölder and the AG mean inequalities, from (3.14) we have

$$
\begin{aligned}
\Gamma(z, t) \leq & m_{1}(t)\left(-\frac{\partial \Gamma}{\partial z}(z, t)\right)+\frac{\beta^{2}}{2 K^{*} m_{1}(t) \lambda_{1}} \int_{0}^{t} \int_{\boldsymbol{R}_{z}} \boldsymbol{T}_{, i} \boldsymbol{T}_{, i} \mathrm{~d} x \mathrm{~d} \eta \\
& +\frac{m_{1}(t) K^{*}}{2} \int_{0}^{t} \int_{\boldsymbol{R}_{z}} \theta_{, i} \theta_{, i} \mathrm{~d} x \mathrm{~d} \eta+\frac{\beta^{2}}{2 K^{*}} \int_{0}^{t} \int_{\boldsymbol{R}_{z}}(\xi-z) \boldsymbol{T}_{, i} \boldsymbol{T}_{, i} \mathrm{~d} x \mathrm{~d} \eta \\
& +\frac{K^{*}}{2} \int_{0}^{t} \int_{\boldsymbol{R}_{\mathrm{Z}}}(\xi-z) \theta_{, i} \theta_{, i} \mathrm{~d} x \mathrm{~d} \eta .
\end{aligned}
$$

In view of the definitions of the functions $\Phi(z, t)$ and $\Psi(z, t)$, we introduce the functions

$$
\begin{aligned}
\tilde{\boldsymbol{\Phi}}(z, t)= & \frac{\rho}{2} \int_{\boldsymbol{R}_{z}} \dot{w}_{i} \dot{w}_{i} \mathrm{~d} x+\frac{v}{2} \int_{\boldsymbol{R}_{z}} w_{i, j} w_{i, j} \mathrm{~d} x+\frac{\lambda+v}{2} \int_{\boldsymbol{R}_{z}} w_{i, i}^{2} \mathrm{~d} x \\
& +\frac{K^{*}}{2} \int_{0}^{t} \int_{\boldsymbol{R}_{z}} \theta_{, i} \theta_{, i} \mathrm{~d} x \mathrm{~d} \eta+M \int_{0}^{t} \int_{\boldsymbol{R}_{z}} \Sigma_{, i} \Sigma_{, i} \mathrm{~d} x \mathrm{~d} \eta \\
& +\frac{1}{2} \int_{\boldsymbol{R}_{z}}\left[c \theta^{2}+2 d \theta \Sigma+n \Sigma^{2}\right] \mathrm{d} x \mathrm{~d} \eta,
\end{aligned}
$$

and

$$
\begin{aligned}
\tilde{\Gamma}(z, t)= & \int_{z}^{\infty} \tilde{\Phi}(\xi, t) \mathrm{d} \xi \\
= & \frac{\rho}{2} \int_{R_{z}}(\xi-z) \dot{w}_{i} \dot{w}_{i} \mathrm{~d} x+\frac{v}{2} \int_{R_{z}}(\xi-z) w_{i, j} w_{i, j} \mathrm{~d} x \\
& +\frac{\lambda+v}{2} \int_{R_{z}}(\xi-z) w_{i, i}^{2} \mathrm{~d} x+\frac{K^{*}}{2} \int_{0}^{t} \int_{R_{z}}(\xi-z) \theta_{, i} \theta_{, i} \mathrm{~d} x \mathrm{~d} \eta \\
& +M \int_{0}^{t} \int_{R_{z}}(\xi-z) \Sigma_{, i} \Sigma_{, i} \mathrm{~d} x \mathrm{~d} \eta \\
& +\frac{1}{2} \int_{R_{z}}(\xi-z)\left[c \theta^{2}+2 d \theta \Sigma+n \Sigma^{2}\right] \mathrm{d} x \mathrm{~d} \eta .
\end{aligned}
$$

Then, inequality (3.15) may be rewritten as

$$
\begin{aligned}
\tilde{\Gamma}(z, t) \leq & 3 m_{1}(t)\left(-\frac{\partial \tilde{\Gamma}}{\partial z}(z, t)\right)+\frac{\beta^{2}}{2 K^{*} m_{1}(t) \lambda_{1}} \int_{0}^{t} \int_{\boldsymbol{R}_{z}} T_{, i} T_{, i} \mathrm{~d} x \mathrm{~d} \eta \\
& +\frac{\beta^{2}}{2 K^{*}} \int_{0}^{t} \int_{\boldsymbol{R}_{z}}(\xi-z) \boldsymbol{T}_{, i} \boldsymbol{T}_{, i} \mathrm{~d} x \mathrm{~d} \eta .
\end{aligned}
$$

Combining (2.1) and (2.2), we know

$$
\int_{0}^{t} \int_{\boldsymbol{R}_{Z}} \boldsymbol{T}_{, i} \boldsymbol{T}_{, i} \mathrm{~d} x \mathrm{~d} \eta \leq \frac{1}{K} \boldsymbol{E}(0, t) \mathrm{e}^{-\frac{1}{m_{1}(t)} z} .
$$

If we set

$$
\hat{\boldsymbol{E}}(z, t)=\int_{z}^{\infty} \boldsymbol{E}(\xi, t) \mathrm{d} \xi,
$$

from (2.2), we have

$$
\hat{\boldsymbol{E}}(z, t) \leq m_{1}(t) \boldsymbol{E}(0, t) \mathrm{e}^{-\frac{1}{m_{1}(t)}} .
$$


From the definition of $\boldsymbol{E}(z, t)$ and (3.20), we have

$$
\begin{aligned}
\hat{\boldsymbol{E}}(z, t)= & \frac{1}{2} \rho \int_{\boldsymbol{R}_{z}}(\xi-z) \dot{u}_{i} \dot{u}_{i} \mathrm{~d} x+\frac{v}{2} \int_{\boldsymbol{R}_{z}}(\xi-z) u_{i, j} u_{i, j} \mathrm{~d} x \\
& +\frac{\lambda+v}{2} \int_{\boldsymbol{R}_{z}}(\xi-z) u_{i, i}^{2} \mathrm{~d} x+K \int_{0}^{t} \int_{\boldsymbol{R}_{z}}(\xi-z) \boldsymbol{T}_{, j} \boldsymbol{T}_{, j} \mathrm{~d} A \mathrm{~d} \eta \\
& +M \int_{0}^{t} \int_{\boldsymbol{R}_{z}}(\xi-z) \boldsymbol{C}_{, j} \boldsymbol{C}_{, j} \mathrm{~d} A \mathrm{~d} \eta+\int_{\boldsymbol{R}_{z}}(\xi-z)\left[\frac{\boldsymbol{C}}{2} \boldsymbol{T}^{2}+d \boldsymbol{C} \boldsymbol{T}+\frac{n}{2} \boldsymbol{C}^{2}\right] \mathrm{d} x .
\end{aligned}
$$

So, we have the following inequality

$$
\int_{0}^{t} \int_{\boldsymbol{R}_{z}}(\xi-z) \boldsymbol{T}_{, j} \boldsymbol{T}_{, j} \mathrm{~d} A \mathrm{~d} \eta \leq \frac{1}{K} m_{1}(t) \boldsymbol{E}(0, t) \mathrm{e}^{-\frac{1}{m_{1}(t)}} .
$$

Inserting (3.19) and (3.23) back into (3.18), we have

$$
\tilde{\Gamma}(z, t) \leq m_{1}(t)\left(-\frac{\partial \tilde{\Gamma}}{\partial z}(z, t)\right)+\beta^{2} m_{3}(t) \mathrm{e}^{-\frac{1}{m_{1}(t)} z},
$$

where

$$
m_{3}(t)=\frac{\boldsymbol{E}(0, t)}{2 K K^{*} m_{1}(t) \lambda_{1}}+\frac{\boldsymbol{E}(0, t)}{2 K K^{*}} m_{1}(t)
$$

For any fixed $t_{1}>0$, setting $k_{1}=3 m_{1}(t)$ and $k_{2}=\frac{1}{m_{1}(t)}$, from (3.24) we obtain

$$
\frac{\partial \tilde{\Phi}}{\partial z}\left(z, t_{1}\right)+\int_{z}^{\infty} \tilde{\Phi}\left(\xi, t_{1}\right) \mathrm{d} \xi \leq k_{1} \tilde{\Phi}\left(z, t_{1}\right)+\beta^{2} m_{3} \mathrm{e}^{-k_{2} z},
$$

where we have used the fact $\frac{\partial \tilde{\Phi}}{\partial z}\left(z, t_{1}\right)<0$. To get the result we want, we let

$$
\Pi\left(z, t_{1}\right)=\mathrm{e}^{-k_{1} z} \tilde{\boldsymbol{\Phi}}\left(z, t_{1}\right)+h \int_{z}^{\infty} \mathrm{e}^{-k_{1} \xi} \tilde{\boldsymbol{\Phi}}\left(\xi, t_{1}\right) \mathrm{d} \xi .
$$

Thus, inequality (3.26) may be rewritten as

$$
\frac{\partial \Pi}{\partial z}\left(z, t_{1}\right)+h \Pi\left(z, t_{1}\right) \leq \beta^{2} m_{3} \mathrm{e}^{-\left(k_{1}+k_{2}\right) z},
$$

provided $h$ satisfies the quadratic equation

$$
h^{2}-k_{1} h-1=0 .
$$

We make the choice of

$$
h=h_{0}=\frac{k_{1}+\sqrt{k_{1}^{2}+4}}{2} .
$$

For this choice of $h$, to integrate (3.28), we have to consider the following two cases:

1) If $h_{0}-k_{1}-k_{2}=0$, an integration of (3.28) leads to

$$
\Pi\left(z, t_{1}\right) \leq \Pi\left(0, t_{1}\right) \mathrm{e}^{-h_{0} z}+\beta^{2} m_{3} z \mathrm{e}^{-h_{0} z} .
$$

In light of (3.27), we have

$$
\tilde{\boldsymbol{\Phi}}\left(z, t_{1}\right) \leq \Pi\left(0, t_{1}\right) \mathrm{e}^{-\left(h_{0}-k_{1}\right) z}+\beta^{2} m_{3} \mathrm{e}^{-\left(h_{0}-k_{1}\right) z} .
$$

2) If $h_{0}-k_{1}-k_{2} \neq 0$, an integration of (3.28) leads to 


$$
\Pi\left(z, t_{1}\right) \leq \Pi\left(0, t_{1}\right) \mathrm{e}^{-h_{0} z}+\frac{\beta^{2} m_{3}}{h_{0}-k_{1}-k_{2}}\left[\mathrm{e}^{\left(h_{0}-k_{1}-k_{2}\right) z}-1\right] \mathrm{e}^{-h_{0} z}
$$

It is easy to proof that the second term on the right of (3.33) is positive either $h_{0}-k_{1}-k_{2}>0$ or $h_{0}-k_{1}-k_{2}<0$. In view of (3.27), we have

$$
\tilde{\Phi}\left(z, t_{1}\right) \leq\left[\Pi\left(0, t_{1}\right)-\frac{\beta^{2} m_{3}}{h_{0}-k_{1}-k_{2}}\right] \mathrm{e}^{-\left(h_{0}-k_{1}\right) z}+\frac{\beta^{2} m_{3}}{h_{0}-k_{1}-k_{2}} \mathrm{e}^{-k_{2} z} .
$$

In order to make inequalities (3.32) and (3.34) explicit, we need bound for $\Pi\left(0, t_{1}\right)$. From the definition of $\Pi\left(z, t_{1}\right)$ in (3.27), we may write

$$
\Pi\left(0, t_{1}\right)=\tilde{\boldsymbol{\Phi}}\left(0, t_{1}\right)+h \int_{0}^{\infty} \mathrm{e}^{-k_{1} \xi} \tilde{\boldsymbol{\Phi}}\left(\xi, t_{1}\right) \mathrm{d} \xi \leq \tilde{\boldsymbol{\Phi}}\left(0, t_{1}\right)+h \tilde{\boldsymbol{\Gamma}}\left(0, t_{1}\right) .
$$

From (3.35), to bound $\Pi\left(0, t_{1}\right)$ we only need to bound $\tilde{\Phi}\left(0, t_{1}\right)$ and $\tilde{\Psi}\left(0, t_{1}\right)$. From (3.11), we have

$$
\Phi\left(0, t_{1}\right)=-\beta \int_{0}^{t} \int_{R} \boldsymbol{T}_{, i} \theta_{, i} \mathrm{~d} x \mathrm{~d} \eta \leq \frac{\beta^{2}}{2 K^{*}} \int_{0}^{t} \int_{R} \boldsymbol{T}_{, i} \boldsymbol{T}_{, i} \mathrm{~d} x \mathrm{~d} \eta+\frac{K^{*}}{2} \int_{0}^{t} \int_{R} \theta_{, i} \theta_{, i} \mathrm{~d} x \mathrm{~d} \eta
$$

Using (3.12) and (3.16), we have

$$
\tilde{\boldsymbol{\Phi}}\left(0, t_{1}\right) \leq \frac{\beta^{2}}{2 K K^{*}} \boldsymbol{E}\left(0, t_{1}\right) .
$$

From (3.24) and using (3.37), we can get

$$
\tilde{\boldsymbol{\Psi}}\left(0, t_{1}\right) \leq \frac{m_{1} \beta^{2}}{2 K K^{*}} \boldsymbol{E}\left(0, t_{1}\right) .
$$

Combining (3.35), (3.37) and (3.38), we have

$$
\Pi\left(0, t_{1}\right) \leq \beta^{2} m_{4},
$$

where $m_{4}=\frac{\boldsymbol{E}\left(0, t_{1}\right)}{2 K K^{*}}+\frac{h m_{1} \boldsymbol{E}\left(0, t_{1}\right)}{2 K K^{*}}$. Combining the above discussions, we can conclude:

If $h_{0}-k_{1}-k_{2}=0$, we have

$$
\tilde{\Phi}\left(z, t_{1}\right) \leq \beta^{2} m_{4} \mathrm{e}^{-\left(h_{0}-k_{1}\right) z}+\beta^{2} m_{3} \mathrm{e}^{-\left(h_{0}-k_{1}\right) z} .
$$

If $h_{0}-k_{1}-k_{2} \neq 0$, we have

$$
\tilde{\Phi}\left(z, t_{1}\right) \leq \beta^{2}\left[m_{4}-\frac{m_{3}}{h_{0}-k_{1}-k_{2}}\right] \mathrm{e}^{-\left(h_{0}-k_{1}\right) z}+\frac{\beta^{2} m_{3}}{h_{0}-k_{1}-k_{2}} \mathrm{e}^{-k_{2} z} .
$$

Inequalities (3.40) and (3.41) exhibit not only exponential decay in $z$, but also show that the amplitude terms in (3.40) and (3.41) become small as $K \rightarrow K^{*}$.

\section{Conclusion}

In view of the Equations (1.2) and (1.3), we may also obtain the continuous dependence on the coefficient $M$ by employing the methods which have been used in Section 3. Our method is also valid to study other equations. In the future, we will use the method proposed in this paper to study the structural stability for the fluid flow in porous media. We think we will get some interesting results. 


\section{Funding}

The work was supported by the science foundation of Huashang College Guangdong University of Finance \& Economics (Grant \# 2019HSDS28).

\section{Conflicts of Interest}

The author declares no conflicts of interest regarding the publication of this paper.

\section{References}

[1] Ames, K.A. and Straughan, B. (1997) Non-Standard and Improperly Posed Problems. Academic Press, Cambridge.

[2] Straughan, B. (2004) The Energy Method, Stability and Nonlinear Convection. 2nd Edition, Springer-Verlag, New York. https://doi.org/10.1007/978-0-387-21740-6

[3] Celebi, A.O., Kalantarov, V.K. and Ugurlu, D. (2006) On Continuous Dependence on Coefficients of the Brinkman-Forchheimer Equations. Applied Mathematics Letters, 19, 801-807. https://doi.org/10.1016/j.aml.2005.11.002

[4] Payne, L.E. and Straughan, B. (1999) Convergence and Continuous Dependence for the Brinkman-Forchheimer Equations. Studies in Applied Mathematics, 102, 419-439. https://doi.org/10.1111/1467-9590.00116

[5] Payne, L.E. and Straughan, B. (1998) Structural Stability for the Darcy Equations of Flow in Porous Media. Proceedings of the Royal Society A, 454, 1691-1698. https://doi.org/10.1098/rspa.1998.0227

[6] Quintanilla, R. (2003) Convergence and Structural Stability in Thermolasticity. Applied Mathematics and Computation, 135, 287-300. https://doi.org/10.1016/S0096-3003(01)00331-9

[7] Payne, L.E., Song, J.C. and Straughan, B. (1999) Continuous Dependence and Convergence Results for Brinkman and Forchheimer Models with Variable Viscosity. Proceedings of the Royal Society A, 45, 2173-2190. https://doi.org/10.1098/rspa.1999.0398

[8] Payne, L.E. and Straughan, B. (1996) Stability in the Initial-Time Geometry Problem for the Brinkman and Darcy Equations of Flow in Porous Media. Journal de Mathématiques Pures et Appliquées, 75, 225-271.

[9] Nowacki, W. (1971) Certain Problem of Thermodiffusion in Solids. Archives of Mechanics, 23, 731-754.

[10] Nowacki, W. (1994) Dynamical Problems of Thermodiffusion in Solids II. Bulletin of the Polish Academy of Sciences: Technical Sciences, 22, 205-211.

[11] Fichera, G. (1974) Uniqueness, Existence and Estimates of Solution in the Dynamical Problem of Thermodiffusion in an Elastic Solid. Archives of Mechanics, 26, 903-920.

[12] Podstrigach, Y.S., Akad, D. and Ukr, N. (1961) Differential Equations of the Problem of Thermodiffusion in an Isotropic Deformable Solid. Dopovidi Akademiï Nauk Ukraïns' koï RSR, 2, 169-172.

[13] Gawinecki, J.A. and Sierpiński, K. (1982) Existence, Uniqueness and Regularity of the Solution of the First Boundary-Initial Value Problem for the Equation of Thermodiffusion in a Solid Body. Bulletin of the Polish Academy of Sciences. Technical Sciences, 30, 541-547.

[14] Szymaniec, A. (2010) $L^{p}-L^{q}$ Time Decay Estimates for the Solution of the Linear 
Partial Differential Equations of Thermos-Diffusion. Applications Mathematicae, 37, 143-1702. https://doi.org/10.4064/am37-2-2

[15] Kaloni, P.N. and Qin, Y. (1998) Spatial Decay Estimates for Flow in the Brinkman-Forchheimer Model. Quartely of Applied Mathematics, 56, 71-87. https://doi.org/10.1090/qam/1604880

[16] Horgan, C.O. and Wheeler, L.T. (1978) Spatial Decay Estimates for the Navier? Cstokes Equations with Application to the Problem of Entry Flow. SIAM Journal on Applied Mathematics, 35, 97-116. https://doi.org/10.1137/0135008

[17] Payne, L.E. and Song, J.C. (1997) Spatial Decay Estimates for the Brinkman and Darcy Flows in a Semi-Infinite Cylinder. Continuum Mechanics and Thermodynamics, 9, 175-190. https://doi.org/10.1007/s001610050064

[18] Ames, K.A. and Song, J.C. (2006) Decay Bounds for Magnetohydrodynamic Geophysical Flow. Nonlinear Analysis. Theory, Methods \& Applications, 65, 1318-1333. https://doi.org/10.1016/j.na.2005.10.013

[19] Ames, K.A. and Payne, L.E. (1989) Decay Estimates in Steady Pipe Flow. SIAM Journal on Mathematical Analysis, 20, 789-815. https://doi.org/10.1137/0520056

[20] Horgan, C.O. (1996) Recent Developments Concerning Saint-Venants' Principle: A Second Update. Applied Mechanics Reviews, 49, S101-S111. https://doi.org/10.1115/1.3101961

[21] Lin, C. (1992) Spatial Decay Estimates and Energy Bounds for the Stokes Flow Equation. Stability and App Anal of Continuous Media, 2, 249-264.

[22] Lin, C. and Payne, L.E. (2004) Spatial Decay Bounds in the Channel Flow of an Incompressible Viscous Fluid. Mathematical Models and Methods in Applied Sciences, 14, 795-818. https://doi.org/10.1142/S0218202504003453

[23] Song, J.C. (2003) Improved Decay Estimates in Time-Dependent Stokes Flow. SIAM Journal on Mathematical Analysis, 288, 505-517. https://doi.org/10.1016/j.jmaa.2003.09.007

[24] Li, Y. and Lin, C. (2014) Continuous Dependence for the Nonhomogeneous Brinkman-Forchheimer Equations in a Semi-Nfinite Pipe. Applied Mathematics and Computation, 244, 201-208. https://doi.org/10.1016/j.amc.2014.06.082

[25] Liu, Y. (2012) Convergence Results for Forchheimer's Equations. European Journal of Applied Mathematics, 23, 761-775.

[26] Liu, Y. (2017) Continuous Dependence for a Thermal Convection Model with Temperature Dependent Solubility. Applied Mathematics and Computation, 308, 18-30. https://doi.org/10.1016/j.amc.2017.03.004

[27] Liu, Y. and Xiao, S.Z. (2018) Structural Stability for the Brinkman Fluid Interfacing with a Darcy Fluid in an Unbounded Domain. Nonlinear Analysis. Real World Applications, 42, 308-333. https://doi.org/10.1016/j.nonrwa.2018.01.007

[28] Liu, Y., Xiao, S.Z. and Lin, Y.W. (2018) Continuous Dependence for the Brinkman-Forchheimer Fluid Interfacing with a Darcy Fluid in a Bounded Domain. Mathematics and Computers in Simulation, 150, 66-82. https://doi.org/10.1016/j.matcom.2018.02.009

[29] Payne, L.E. (1967) Isopermetric Inequalities and Their Applications. SIAM Review, 9, 453-488. https://doi.org/10.1137/1009070

[30] Pólya, G. and Szegö, G. (1951) Isopermetric Inequalities in Mathematical Physics. Princeton University Press, Princeton. https://doi.org/10.1515/9781400882663

[31] Cai, Z.H. (2020) Spatial Decay Estimates for a Class of Thermo-Diffusion Equations. Pure Mathematics, 10, 265-271. https://doi.org/10.12677/PM.2020.104034 